\title{
A genome scan for loci linked to quantitative insulin traits in persons without diabetes: the Framingham Offspring Study
}

\author{
C. I. M. Panhuysen'1, L. A. Cupples ${ }^{1}$, P. W. F. Wilson' ${ }^{2,}$, A. G. Herbert ${ }^{4}$, R. H. Myers ${ }^{4}$, J. B. Meigs ${ }^{5}$ \\ ${ }^{1}$ Department of Biostatistics, Boston University School of Public Health and School of Medicine, Boston, MA, USA \\ ${ }^{2}$ Framingham Heart Study, Framingham, MA, USA \\ ${ }^{3}$ Boston University School of Medicine, Boston, MA, USA \\ ${ }^{4}$ Department of Neurology, Boston University School of Medicine, Boston, MA, USA \\ ${ }^{5}$ General Internal Medicine and Clinical Epidemiology Units, General Medicine Division, Department of Medicine, \\ Massachusetts General Hospital and Harvard Medical School, Boston, MA, USA
}

\section{Abstract}

Aims/hypothesis. Hyperinsulinaemia and insulin resistance usually precede clinical hyperglycaemia and Type 2 diabetes. Thus, plasma insulin concentrations and insulin resistance are important quantitative traits associated with risk of Type 2 diabetes, and represent key measures for genetic analysis of the syndrome.

Methods. We carried out a genome-wide search for loci related to plasma insulin concentrations and insulin resistance in 330 extended, community-based pedigrees from the Framingham Heart Study. Normalized deviates of the standardized residuals of plasma insulin concentrations in the fasting state, $2 \mathrm{~h}$ after oral glucose challenge and as a measure of insulin resistance were used in linkage analysis with the variance components model implemented in the computer program SOLAR. Results. The results suggest susceptibility loci influencing plasma concentrations of fasting insulin and insulin resistance on chromosomes 11 (LOD 2.43 at $85 \mathrm{cM}$ close to D11S2002) and 17 (LOD 1.8 at $60 \mathrm{cM}$, close to D17S784); and susceptibility loci influencing 2-h plasma insulin concentrations on chromosomes 9 (LOD 2.8 at $80 \mathrm{cM}$, close to D9S922) and 19 (LOD 1.8 at $66 \mathrm{cM}$, close to D19S245). The results of the analysis of 1000 simulations of the trait and an unlinked marker suggest that in a genome scan of 401 markers fewer than one LOD score over 1 would be due to Type 1 error, and be a false positive.

Conclusion/interpretation. We conclude that these suggestive regions for quantitative pre-diabetic insulin traits could contain major loci in the pathogenesis of Type 2 diabetes. [Diabetologia (2003) 46:579-587]

Keywords Insulin, insulin resistance, diabetes mellitus, non-insulin-dependent, chromosome mapping, chromosomes, human, pair 9, chromosomes, human, pair 11, chromosomes, human, pair 17.
Received: 4 June 2002 / Revised: 4 November 2002

Published online: 21 March 2003

C) Springer-Verlag 2003

Corresponding author: J. B. Meigs, General Internal Medicine and Clinical Epidemiology Units, General Medicine Division, Department of Medicine, Massachusetts General Hospital and Harvard Medical School, 50 Staniford St. 9th Floor, Boston, 02114 MA., USA

E-mail: jmeigs@partners.org

Abbreviations: DM, Diabetes mellitus; IR, Insulin resistance; HOMA-IR, homeostasis model-assessed insulin resistance (fasting insulin $(\mathrm{uU} / \mathrm{ml}) \times$ fasting glucose $(\mathrm{mmol} / \mathrm{l})) / 22.5$; LOD, logarithm of the odds ratio of linkage between a chromosomal locus and a trait; NPL, non-parametric LOD score; QTL, quantitative trait locus.
Type 2 diabetes is a common metabolic disorder affecting about 6 to $7 \%$ of the US population and is increasingly common worldwide. It is defined by high plasma concentrations of plasma glucose in either the fasting state or after an oral glucose challenge [1]. Development of hyperglycaemia and Type 2 diabetes is characterized by tissue resistance to insulin action and, despite compensatory hyperinsulinaemia, failure of pancreatic beta cells to produce plasma concentrations of insulin adequate to overcome peripheral insulin resistance (IR) [2]. It is widely accepted that IR is an early finding, evident before the onset of hyperglycaemia and predictive of the development of Type 2 diabetes [3]. As such, plasma concentrations of insulin and of IR are important quantitative traits associated with risk for developing Type 2 diabetes, and re- 
present powerful measures in the genetic analysis of the syndrome.

Several genome-wide scans have been carried out on prediabetic insulin traits. In most studies however, the families and participants were collected through an index patient with Type 2 diabetes, and the study cohorts were known to have a higher risk for developing Type 2 diabetes, such as Pima Indians, Mexican Americans and Australian Aborigines. in unaffected individuals three genome scans carried out from such higher risk populations resulted in several candidate regions on chromosome $3[4,5]$, chromosome 4 [4], chromosome 9 [4], chromosome 10 [6], chromosome 13 [6], chromosome 17 [6] and chromosome 22 [4]. However, probably due to the complex nature of the disease, failure of replication of postulated genes and false-positive discovery of new loci remains a major concern.

We provide results of a genome-wide search for loci associated with plasma concentrations of insulin and IR (expressed as homeostasis model-assessed IR, or HOMA-IR) from an unselected, low diabetes-risk cohort including 330 extended, community-based pedigrees from the Framingham Heart Study [7].

\section{Subjects and Methods}

Subjects. Participants of the Framingham Offspring Study are the natural children of the original Framingham Heart Study families. At the first Offspring Study examination from 1971 to 1975 participant ages ranged from 12 to 58 years. In 1995, at the 5th examination, 2607 offspring in 1643 pedigrees completed 20 years of quadrennial follow-up, with the exception of 8 years between the first and second exams. These subjects underwent a clinical and fasting laboratory examination, with written informed consent obtained prior to examination. The Boston University Institutional Review Board approved the study for Human Subjects Research.

In this analysis we included members of 330 extended pedigrees, consisting of 1444 nuclear families, (original Cohort parents with offspring), in whom 1815 individuals were genotyped by the Marshfield Mammalian Genotyping Service. Of these, 113 individuals with familial or genotyping errors were excluded, leaving 1702 individuals accurately genotyped, of whom 394 were original cohort members and 1308 were offspring study subjects.

Among offspring subjects with genotype data, 1214 subjects (600 men and 614 women) attended exam 5 and provided phenotype data. Due to missing values in the independent parameters used in adjustment of insulin traits, the final analysis included 1158, 1134, and 1157 offspring subjects contributing data on plasma concentrations of fasting insulin, insulin at $2 \mathrm{~h}$ after an oral glucose challenge (2-h insulin), and HOMA-IR respectively. Although phenotypic trait data were not available from original cohort subjects, 394 of them contributed genotypic information to the IBD sharing probabilities in this analysis.

Subjects previously diagnosed and treated for diabetes were excluded from the analysis, since diabetes treatment directly affects the insulin measures and could cause subjects who are carriers of one or more diabetes susceptibility loci to have insulin plasma concentrations within the normal range, thereby masking evidence for linkage. Diabetes was defined by self-reported hypoglycaemic drug therapy or by two or more fasting plasma glucose concentrations greater than or equal to $7.0 \mathrm{mmol} / \mathrm{l}$ at any exam (1-5) [8].

Covariates. Body mass index (BMI) was calculated as weight in kilograms divided by the square of height in meters $\left(\mathrm{kg} / \mathrm{m}^{2}\right)$. Smoking was assessed as the number of cigarettes smoked on average per day during the year before the examination (cigs/day). Physical activity was assessed as a weighted sum of the number of hours of a typical day spent sleeping and doing sedentary, slight, moderate, or heavy physical activities [9]. Alcohol use was categorized as usual consumption in ounces of alcohol per week (oz/wk). Estrogen use (either birth control or estrogen replacement therapy) was defined as present or absent.

Metabolic testing. Fasting insulin was measured in EDTA plasma as total immunoreactive insulin (Coat-A-Count Insulin, Diagnostic Products, Los Angeles, Calif., USA). Cross-reactivity of this assay with pro-insulin at mid-curve is approximately $40 \%$, the intra-assay and inter-assay CV ranged from 5.0\% to $10.0 \%$ for concentrations reported here, and the lower limit of sensitivity was $1.1 \mu \mathrm{U} / \mathrm{ml}(8 \mathrm{pmol} / \mathrm{l})$. Plasma glucose was measured with a hexokinase reagent kit (A-gent glucose test, Abbott, South Pasadena, Calif., USA). Glucose assays were run in duplicate and the intra-assay coefficient of variation ranged from $2 \%$ to $3 \%$, depending on the assayed glucose concentration.

HOMA-IR was calculated as [fasting plasma insulin $(\mathrm{uU} / \mathrm{ml}) \times$ fasting plasma glucose $(\mathrm{mmol} / \mathrm{l}) / 22.5]$ [10].

Concentrations of fasting insulin and HOMA-IR both provide reliable estimates of IR across the range of glucose tolerance using either the frequently-sampled intravenous glucose tolerance test with minimal model analysis or the glucose clamp technique as the gold-standard measure of insulin sensitivity [11, 12]. Fasting hyperinsulinaemia and increased HOMA-IR are powerful predictors of increased risk for glucose intolerance [13, 14, 15]. Hyperinsulinaemia $2 \mathrm{~h}$ after an oral glucose challenge is a characteristic of impaired glucose tolerance and also confers increased risk for Type 2 diabetes [13]. As such, the insulin measures considered here represent valid quantitative traits for genetic analysis of Type 2 diabetes.

Statistical methods. Descriptive statistics were computed using means and standard deviations for continuous measurements. A $p$ value less than 0.05 was considered statistically significant. Linear regression models were used prior to linkage analyses to adjust the insulin traits for potentially confounding covariates, including age, smoking, alcohol use, physical activity and estrogen usage in women. Since there is a known difference in these confounders between genders separate regression models were used for men and women. The residuals from these models thus reflect deviations from the mean value for subjects in the cohort with similar covariates. These residuals were used for the linkage analyses with men and women combined. Separate models were constructed with or without a term for BMI.

Linkage analyses were carried out on the normalized deviates derived from the ranked standardized residuals for concentrations of fasting insulin, 2-h insulin and HOMA-IR to avoid the potential for false positives induced by analysis of the skewed data distributions. We constructed models with and without covariate adjustment and with and without adjustment for BMI in order to show the effect of phenotype manipulation on residual polygenic variance. Models not including BMI were run based on the hypothesis that obesity might be in the causal pathway leading to Type 2 diabetes. In that situation adjusting for values of BMI would obscure evidence for linkage to a specific locus. 
Table 1. Characteristics of all offspring subjects included in the analysis

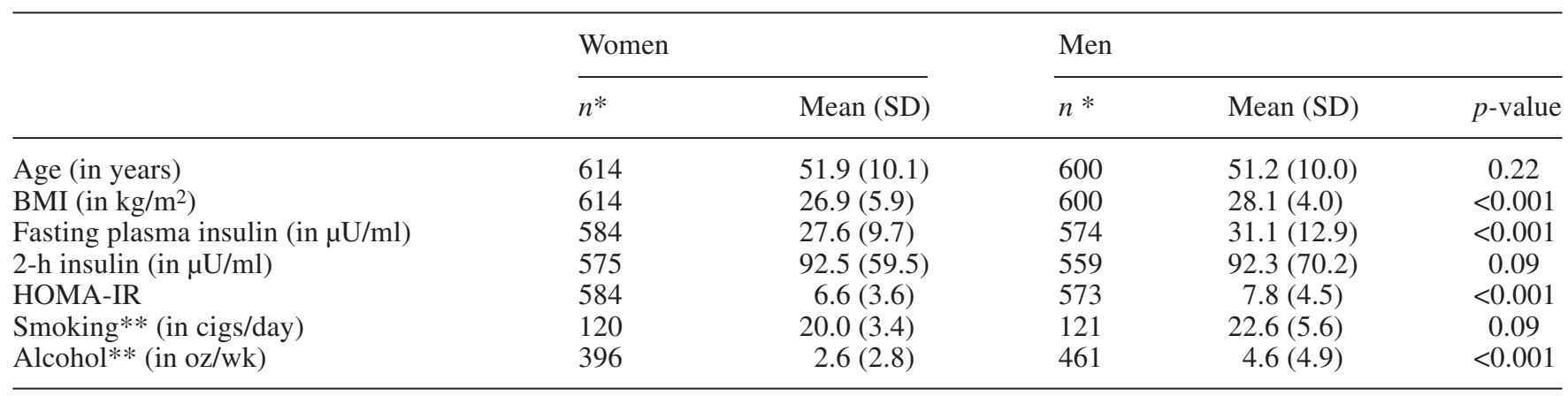

* $n$ : number of individuals, SD: standard deviation

** Self-reported; only subjects responding affirmative were listed

Genotyping. Genomic DNA was extracted from peripheral lymphocytes using a Qiagen Blood and Cell Culture DNA Maxi Kit (Qiagen, Valencia, Calif., USA). The Marshfield Mammalian Genotyping Service carried out a genome-wide scan. The set of 401 microsatellite markers [16] covers the genome at an average density of one marker every $10 \mathrm{cM}$ and has an average heterozygosity of 0.77 (Screening Set v. 8) [17]. The screening set and genotyping protocols are available at the website of the Center for Medical Genetics, Marshfield Medical Research Foundation (http://research.marshfieldclinic.org/genetics/). Map distances were assumed according to the Marshfield map.

Genetic analysis. Familial relationships were checked using ASPEX, and random genotyping errors were checked using Gentest, a precursor to the INFER procedure, in PEDSYS. We carried out linkage analysis using the variance component model implemented in SOLAR (Sequential Oligogenic Linkage Analysis Routines) for quantitative traits. As the insulin quantitative trait distributions were skewed and the variance component method is sensitive to deviations from the normal distribution, we obtained normalized deviates from the standardized residuals from multivariate linear regression models, thereby forming perfectly normally distributed measures. The variance component model in SOLAR makes no assumptions about the mode of transmission and uses all of the data from the pedigree without breaking it up into smaller subsets [18]. The results are expressed in LOD scores, the logarithm of the odds ratio of linkage between a chromosomal locus and a trait. A LOD score of at least 1.9 marks a region with suggestive linkage while a LOD score over 3.3 is considered evidence of genome-wide significance [19].

We further evaluated the potential for obtaining false-positive evidence for linkage by deriving the distribution of nominal LOD scores under the null hypothesis of no linkage by simulation using the SIMQTL script implemented in SOLAR. We simulated an unlinked marker using our pedigree structure and assigned trait values for each derived trait to those subjects in our pedigrees that had the observed traits. Since the pedigree structure for the traits fasting plasma insulin and HOMAIR are almost identical the simulations would be identical too. For this reason only simulations for fasting plasma insulin and for 2-h insulin concentrations were carried out. These newlycreated unlinked data sets were analyzed with a two-point linkage analysis to generate an expected LOD score distribution under the null hypothesis. We repeated this procedure 1000 times for both simulated traits and estimated the proportion of samples that had LOD scores greater than one, two or three. This strategy can be used to evaluate the potential proportion of false positives given the observed pedigree structure.
Table 2. Heritability estimates

\begin{tabular}{llll}
\hline Trait & Heritability & Std Error & $n$ \\
\hline Fasting Insulin & & & \\
- Crude & 0.51 & 0.073 & 1158 \\
- Age, sex adjusted & 0.49 & 0.073 & 1158 \\
- Age, sex and BMI adjusted & 0.48 & 0.073 & 1158 \\
- Fully adjusted w/o BMI* & 0.43 & 0.071 & 1158 \\
- Fully adjusted* & 0.46 & 0.072 & 1158 \\
2-h Insulin & & & \\
- Crude & 0.54 & 0.074 & 1134 \\
- Age, sex adjusted & 0.47 & 0.073 & 1134 \\
- Age, sex and BMI adjusted & 0.47 & 0.072 & 1134 \\
- Fully adjusted w/o BMI* & 0.47 & 0.072 & 1134 \\
- Fully adjusted* & 0.47 & 0.072 & 1134 \\
HOMA-IR & & & \\
- Crude & & & \\
- Age, sex adjusted & 0.52 & 0.074 & 1157 \\
- Age, sex and BMI adjusted & 0.45 & 0.072 & 1157 \\
- Fully adjusted w/o BMI* & 0.42 & 0.073 & 1157 \\
- Fully adjusted* & 0.44 & 0.071 & 1157 \\
\hline
\end{tabular}

* Fully adjusted; insulin traits were adjusted for potentially confounding covariates, including age (with squared and cubic terms to allow for non-linearity), sex, smoking, alcohol use, physical activity, estrogen usage in women. Separate models were constructed with or without a term for BMI

\section{Results}

A total of 330 pedigrees consisting of 1444 nuclear families with 4692 subjects were analyzed (Table 1). Of all subjects fitting the inclusion criteria, 1158 individuals had measures of fasting plasma insulin concentrations, 1134 had 2-h plasma insulin concentrations and from 1157 subjects a HOMA-IR value could be calculated. Phenotype manipulation had a minimal effect on the estimated heritability for all three traits. The heritability was at the most $10 \%$ higher in the ranked crude values as compared to the adjusted traits. For fasting insulin, 2-h insulin and HOMA-IR heritability was 0.4 or greater, suggesting that these traits have a substantial genetic basis (Table 2). 
Table 3. Maximum multipoint LOD scores for normalized deviates of fasting insulin, 2-h insulin and HOMA-IR (not adjusted for $\mathrm{BMI}$ ) listed by chromosome and position in cM. The LOD scores over 1.9 reflecting suggestive linkage are highlighted

\begin{tabular}{|c|c|c|c|c|c|c|c|c|c|}
\hline \multirow[t]{2}{*}{ Chr } & \multicolumn{3}{|c|}{ Fasting Insulin } & \multicolumn{3}{|c|}{ 2-h Insulin } & \multicolumn{3}{|c|}{ HOMA-IR } \\
\hline & LOD & $\mathrm{cM}$ & Close to Marker(s) & LOD & $\mathrm{cM}$ & Close to Marker(s) & LOD & $\mathrm{cM}$ & Close to Marker(s) \\
\hline 2 & 0.8594 & 1 & D2S2976 & 1.0416 & 0 & D2S2976 & 0.6673 & 0 & D2S2976 \\
\hline 3 & 1.1923 & 212 & D3S2398-D3S2418 & 0.313 & 185 & D3S3053-D3S2427 & 1.3019 & 211 & D3S2398 \\
\hline 4 & 0.8231 & 139 & D4S1644 & 0.5663 & 195 & D4S408 & 1.0857 & 136 & D4S2394-D4S1644 \\
\hline 7 & 0.0043 & 105 & D7S821 & 0.0745 & 5 & D7S3056 & 0.0222 & 70 & D7S1818 \\
\hline 8 & 0.2114 & 140 & D8S1128 & 0.6149 & 10 & D8S277 & 0.2432 & 130 & D8S592-D8S1179 \\
\hline 9 & 0.5513 & 65 & D9S301 & 2.8406 & 80 & D9S922 & 0.7469 & 65 & D9S301 \\
\hline 10 & 0.0872 & 155 & D10S1213-D10S1248 & 1.3863 & 70 & D10S1208-D10S1227 & 0.2481 & 155 & D10S1213-D10S1248 \\
\hline 11 & 2.4275 & 88 & D11S2002 & 0.2656 & 105 & D11S1986 & 2.0118 & 92 & D11S2002-D11S2000 \\
\hline 12 & 0.4772 & 40 & D12S373-D12S1042 & 0.0546 & 95 & D12S1064 & 0.3119 & 45 & D12S1042 \\
\hline 17 & $1.8038 *$ & $60 *$ & D17S1299* & 0.8194 & 113 & D17S784 & 2.0453 & 60 & D17S1299 \\
\hline 18 & 0.0371 & 105 & ATA82B02 & 0.0005 & 0 & GATA178F11 & 0.3993 & 105 & ATA82B02 \\
\hline 19 & 0.6571 & 70 & D19S178 & 1.8181 & 66 & D19S178 & 0.6371 & 80 & D19S246 \\
\hline 20 & 0.0759 & 55 & D20S478 & 0.0632 & 65 & D20S481 & 0.0983 & 55 & D20S478 \\
\hline 21 & 1.0558 & 3 & D21S1432 & 0 & 55 & - & 1.1175 & 3 & D21S1432 \\
\hline 22 & 0.1185 & 0 & D22S420 & 1.3386 & 0 & $\mathrm{D} 22 \mathrm{~S} 420$ & 0.086 & 0 & D22S420 \\
\hline
\end{tabular}

* The LOD score of fasting serum insulin concentrations and markers on chromosome 17 only reflect suggestive linkage after adjustment for BMI (multipoint LOD 2.2 at $\sim 66 \mathrm{cM}$ from pter)

A genome scan was done to identify chromosomal regions that might contain genes influencing these traits and maximum multipoint LOD scores for insulin traits by chromosome with the associated marker and map location (Table 3). The regions with suggestive linkage (LOD >1.9) are highlighted. The results of the whole-genome scan for the traits fasting insulin, 2-h insulin and HOMA- (Fig. 1) multipoint linkage analyses on chromosome 11, 17 and 9 are shown (Fig. 2A-C).

On chromosome 11, suggestive evidence was found for linkage to fasting insulin concentrations at about $88 \mathrm{cM}$ from pter (multipoint LOD 2.43, two-point LOD 2.04 at marker D11S2002 at $85.48 \mathrm{cM}$ ). When fasting insulin concentrations were adjusted for BMI, the LOD score dropped from 2.4 to 1.2 and the peak shifted about $10 \mathrm{cM}$, to about $101 \mathrm{cM}$. HOMA-IR showed a peak LOD in the same region (LOD 2.0 at $92 \mathrm{cM}$ ), with a similar decline when adjusted for BMI (Fig. 2A).

The 2-h insulin concentrations had no LOD score exceeding 0.4 on this chromosome.

On chromosome 17 possible evidence was observed for linkage of fasting plasma insulin at about $60 \mathrm{cM}$ from pter (multipoint LOD 1.8, two-point LOD 1.3 at marker D17S974 (GATA8c04)). When adjusted for BMI the multipoint LOD score became more suggestive, increasing to 2.2 while the peak shifted to $66 \mathrm{cM}$. In the same region we found evidence for link- age to HOMA-IR values (multipoint LOD 2.0 at $\sim 60 \mathrm{cM}$ from pter) while after adjustment for BMI the LOD score dropped to 1.8 at $66 \mathrm{cM}$. Plasma 2-h insulin did not show substantial evidence for linkage to chromosome 17.

Two apparent linkage peaks for fasting insulin and HOMA-IR are seen on chromosome 17. The first peak at about $22 \mathrm{cM}$ from pter with a multipoint LOD score of 1.4 (fasting insulin) and 1.2 (HOMA-IR), and the second peak at about 60 from pter for fasting insulin a two-point LOD 1.3 at marker D17S974 (GATA8c04) and with a multipoint LOD score of 1.8 , and for HOMA-IR a multipoint LOD score of 2.0 [two-point LOD 1.5 at marker D17S974 (GATA8c04)], respectively. Although adjusting for BMI did not change the results of the highest peak, it rearranged the general shape of the graph to one peak at about $65 \mathrm{cM}$ with a multipoint LOD score of 2.2 for fasting insulin (Fig. 2B).

On chromosome 9, 2-h plasma insulin showed a multipoint peak LOD score of 2.8 at $80 \mathrm{cM}$ pter while the BMI adjusted values at the same location did not exceed a LOD of 0.35 . The maximum LOD score of fasting insulin and HOMA-IR on chromosome 9 were 0.55 and 0.74 , both at about $65 \mathrm{cM}$ from pter.

On chromosome 19 the multipoint LOD score for 2-h insulin was 1.8 at about $66 \mathrm{cM}$ pter (two-point LOD 2.1), and showed minimal change after BMI adjustment. The maximum LOD score of fasting insulin 
Genome-wide scan for loci linked to fasting insulin concentrations, $2 \mathrm{~h}$ insulin concentrations and levels of HOMA-IR.

Chromosome 1

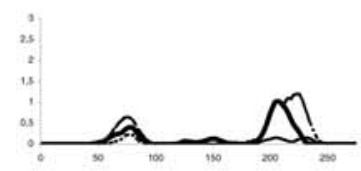

Chromosome 2

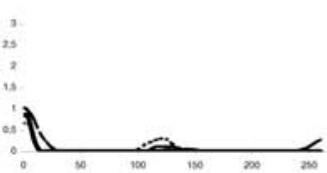

Chromosome 3

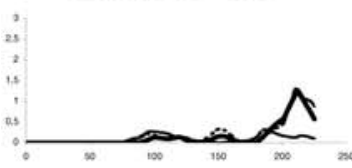

Chromosome 4

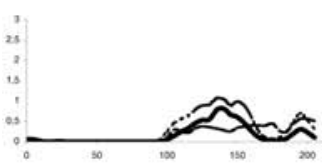

Chromosome 13

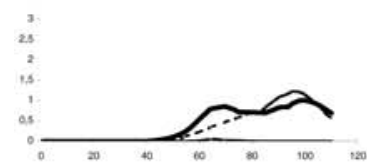

Chromosome 14

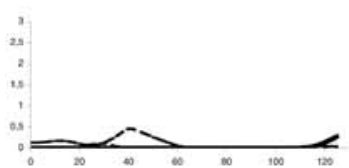

Chromosome 15

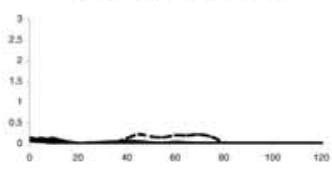

Chromosome 16

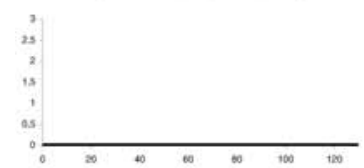

Chromosome 5

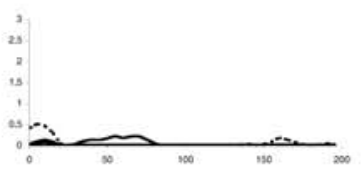

Chromosome 6

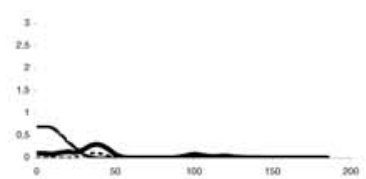

Chromosome 7

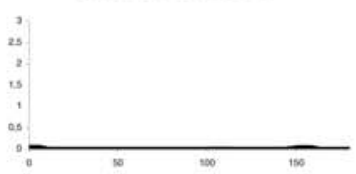

Chromosome 8

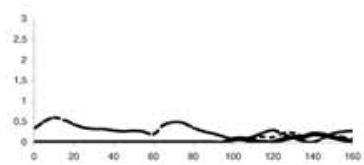

Chromosome 17

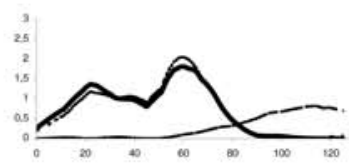

Chromosome 18

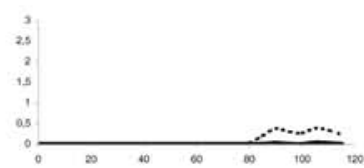

Chromosome 19

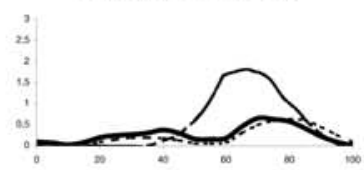

Chromosome 20

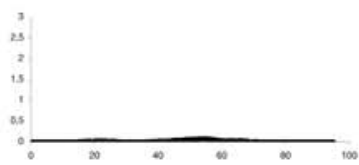

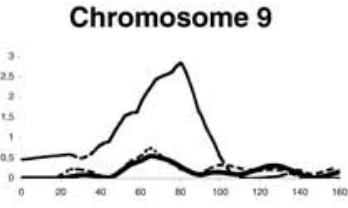

Chromosome 10

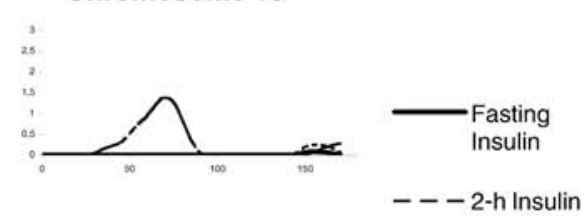

Chromosome 11

.... HOMA-IR

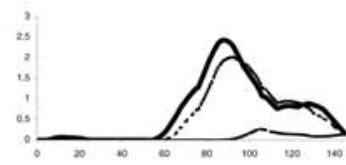

Chromosome 12

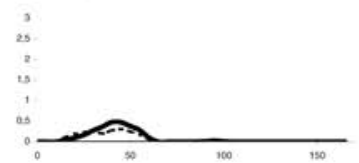

Chromosome 21

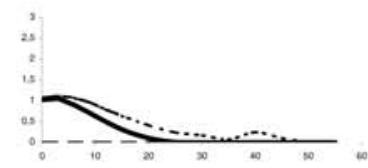

Chromosome 22

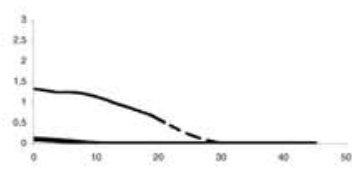

Fig. 1. Genome-wide scan for loci linked to fasting plasma insulin concentrations, 2-h plasma insulin concentrations and values of HOMA-IR 

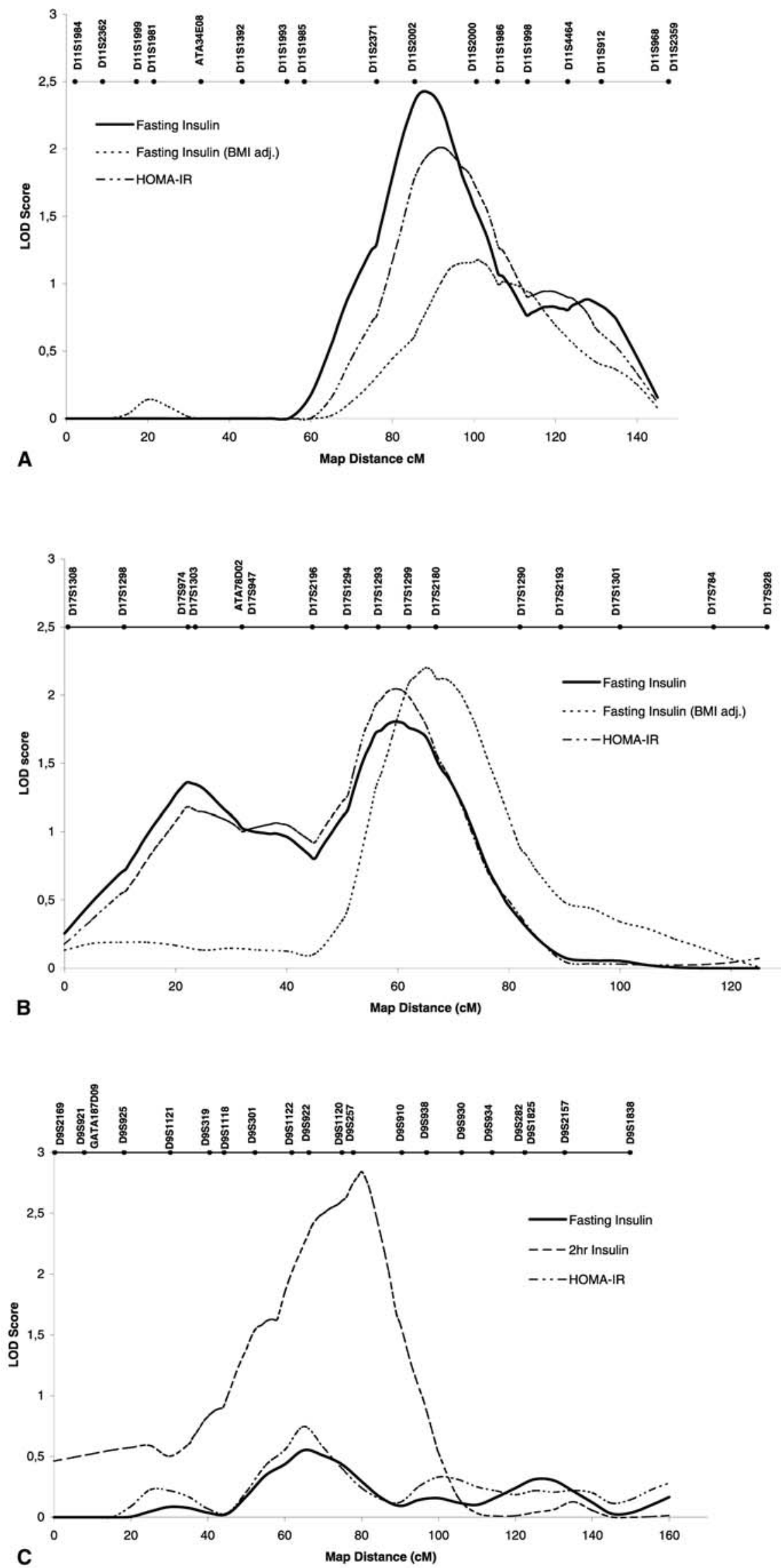
and HOMA-IR on chromosome 19 were 0.66 and 0.63 , at about $65 \mathrm{cM}$ and about $80 \mathrm{cM}$ from pter, respectively (results not shown).

Other potentially interesting regions in which we found a multipoint LOD score exceeding 1.0, but less than 2.0 (Table 3 ) were on chromosome 1 at about 210-230 cM from pter, on chromosome 3 at about 205-220 cM from pter, on chromosome 10 at about 60-80 cM from pter, on chromosome 13 at about 90-100 cM from pter, on chromosome 21 at the p-terminal $0-5 \mathrm{cM}$, and on chromosome 22 at the p-terminal at $0-10 \mathrm{cM}$.

One thousand simulations of an unlinked marker to a simulated trait mimicking fasting insulin concentrations produced a median two-point LOD score of 0 . In $1.9 \%$ of simulations a LOD score greater than 1.0 was obtained (mean 1.34, std.dev. 0.33), of which $0.1 \%$ had a LOD score greater than 2.0 (LOD score 2.05). No LOD score greater than 3.0 was found. For 2-h plasma insulin the simulations yielded a median two-point LOD score of $0.0001 ; 1.5 \%$ of simulations had a LOD score greater than 1.0 (mean 1.36, std.dev. 0.31), and $0.1 \%$ had a LOD score greater than 2.0 (LOD score 2.01). No LOD score greater than 3.0 was found.

\section{Discussion}

Our results suggest the existence of at least two genomic susceptibility loci influencing fasting plasma insulin and two loci influencing the 2-h insulin concentrations in a primarily Caucasian population sample. The results of fasting plasma insulin and insulin resistance estimated by HOMA-IR values were comparable and might reflect effects of the same gene(s). We found the strongest evidence for linkage with fasting plasma insulin and IR at about 88 to $92 \mathrm{cM}$ from pter on chromosome 11. For 2-h plasma insulin the strongest evidence for linkage on chromosome 9 at about $80 \mathrm{cM}$ from pter. These LOD scores decreased dramatically when the traits were adjusted for BMI. This effect of BMI adjustment suggests that the observed linkage between specific genomic loci and insulin traits is mediated in part by overall obesity, as reflected by BMI. This is consistent with studies in diverse populations showing that baseline weight or weight gain over many years increases the risk of incident Type 2 diabetes by $30 \%$ to $400 \%$ [20, 21, 22, 23,

4 Fig. 2. A Multipoint linkage analysis of markers on chromosome 11 with serum concentrations of fasting insulin (adjusted and not adjusted for BMI) and values of HOMA-IR. B Multipoint linkage analysis of markers on chromosome 17 with serum concentrations of fasting insulin (adjusted and not adjusted for BMI) and values of HOMA-IR. C Multipoint linkage analysis of markers on chromosome 9 with serum concentrations of fasting insulin, 2-h insulin concentrations and values of HOMA-IR
24]. It remains uncertain how adipose tissue itself directly augments diabetes risk. Obesity could cause diabetes by inducing IR in the liver and skeletal muscle [25] or by impairing beta-cell function [26], possibly mediated through excessive adipocyte release of free fatty acids, tumour necrosis factor-alpha or other inflammatory cytokines or energy-related hormones. $[27,28,29,30,31]$. By these mechanisms obesity could represent a physiological "environmental" risk factor for Type 2 diabetes, such that BMI adjustment of genetic models could diminish the mediating effect of overall obesity, uncovering the marginal effects of other diabetes-related traits such as hyperinsulinaemia. Thus, obesity could confer a gene-"environment" interactive effect on risk for Type 2 diabetes. In contrast, obesity itself has a genetic basis, [32, 33] and some data suggest that specific loci linked to obesity traits could have pleiotropic effects, as one gene can have multiple, consequences that are seemingly unrelated. In that case the same gene influencing obesity could also affect plasma insulin and susceptibility to Type 2 diabetes [34, 34, 35, 36, 37]. From this perspective adjustment of linkage analyses for BMI could inappropriately obscure evidence for genes that influence other related Type 2 diabetes traits.

On chromosome 11q the evidence for suggestive linkage drops substantially for both traits after BMI adjustment. Several independent studies have reported linkage of BMI to the same area on chromosome 11 [34, 38]. However, in our case, it is unclear whether obesity leads to insulin resistance, or whether hyperinsulinaemia leads to obesity. Intriguingly, the $i d d m 4$ locus for Type 1 diabetes also maps to this region [39], suggesting that a gene in this region could both account for insulin insufficiency in both Type 1 and Type 2 diabetes.

We found similar patterns for 2-h plasma insulin. After adjusting for BMI the evidence for linkage on chromosome 9 ( $~ 80 \mathrm{cM}$, LOD 2.8) disappeared completely ( $\mathrm{LOD}=0.35$ ) while the results on chromosome 19 seemed to be completely indifferent to BMI adjustment. Although high plasma insulin and IR are prediabetic phenotypes, and most likely have a less complicated genetic background than the diabetes as a whole, it is clear from these and other genome scans that BMI plays an unpredictable and poorly-defined role in the expression of some, but not all Type 2 diabetes susceptibility genes. Given the polygenic nature of obesity and Type 2 diabetes it is to be expected that each trait will reflect distinct gene-environment interactions. The regions on chromosomes 11 and chromosome 9 could represent pleiotropic loci, influencing both the insulin traits and BMI. The regions on chromosomes 17 and 19 seem to represent loci more specific to the insulin traits in response to other environmental cues. For example, the peak on chromosome 17 contains the gene for gastric-inhibitory peptide, a hormone that stimulates insulin secretion in response to oral glucose [40]. 
Several other studies have provided similar data on genomic associations with Type 2 diabetes traits. A genome scan on abdominal obesity-metabolic syndrome-related phenotypes in 507 Caucasian families, including plasma insulin concentrations reported two regions of interest, one on chromosome 3 and the other on chromosome $17 \mathrm{p} 12$ [37]. The trait linked to the chromosome 17 region was plasma leptin concentrations and the region was around $38 \mathrm{cM}$ from pter. Interestingly, this seems to correspond with the small peak on our chromosome 17 result of fasting plasma insulin and HOMA-IR (Fig. 2B) that disappears completely when we adjust the traits for BMI.

In a genome scan in non-diabetic Pima Indians [4] a region on chromosome 11 was reported to be linked fasting plasma insulin. This region was the same as we report, but their results lost statistical significance in the multipoint analysis, and the LOD score dropped to 0.25 . In another genome scan of Pima Indians, this time including diabetic subjects, mild linkage of Type 2 diabetes to a region on chromosome 11 was reported, LOD 1.7 (between D11S4464 and D11S912; 123 and $131 \mathrm{cM}$ respectively on the Marshfield map) [34]. Although this peak is not exactly at the same location as ours ( 88-102 cM from pter), the distance is about $35 \mathrm{cM}$ and perhaps distant enough to be considered a separate finding.

In a genome scan in 719 Finnish sib-pairs with Type 2 diabetes an MLS of 1.75 was found in exactly the same region on chromosome $11(84 \mathrm{cM})$ [41]. In a QTL analysis in the same population, however, this region has not been replicated [6]. Other reported findings of linkage between insulin traits and loci on chromosome 11 mostly refer to $11 \mathrm{p}$, like sur 1 at $11 \mathrm{p} 15.1$ at $20-26 \mathrm{cM}$ pter and refer clearly to a different chromosomal region [42].

In an earlier genome scan in non-diabetic Pima Indians [4], several regions linked to 2-h plasma insulin are mentioned, the highest multipoint peak being at 9q21 (LOD 2.16 at $99 \mathrm{cM}$ ) which falls in the same region we found suggestive evidence for linkage with 2-h insulin concentrations (LOD 2.4 at $84 \mathrm{cM}$ ). In a genome-wide scan in 102 Chinese families, for Type 2 diabetes, affected sib-pair analysis revealed two markers on chromosome 9 that showed suggestive evidence, D9S171 $(43 \mathrm{cM})$ and D9S175 $(70 \mathrm{cM})$ with an NPL of 3.3 and 2.9, ( $p$ values 0.00012 and 0.00045 ) respectively [43]. As all these linkage signals give LOD scores below genome-wide significance, replication in other independent cohorts or populations are needed. In this light our findings on chromosomes 11 and 9 are important and strengthen the evidence shown in previously published studies.

Although none of our results exceed a LOD score of 3.3 , the analysis of simulated unlinked markers indicated that our findings are unlikely to be spurious, false positive peaks. For each trait there was a less than 2 in 1000 chance of finding a peak with a two-point LOD of
1.0 or higher. In a genome scan of 401 markers fewer than one LOD score over 1 would be expected to be due to type 1 error, while the expected rate of a LOD score over 2 due to type I error would be close to 0 .

In summary, we found suggestive evidence for linkage of some regions to several insulin traits implicated in the pathogenesis of Type 2 diabetes on chromosomes 9, 11 and 17. Substantial attenuation of linkage signals when plasma insulin concentrations were adjusted for BMI suggest either pleiotropy for both insulinaemia and obesity at these loci, or that overall obesity directly mediates associations of these loci with plasma insulin concentrations. Further work is required to test associations of candidate genes in these regions with occurrence of Type 2 diabetes and/ or obesity, as well as to map other likely diabetes genes to these regions.

Acknowledgements. We thank D.M. Nathan MD and D.E. Singer MD for help with collection and analysis of plasma insulin concentrations and J. Weber PhD and the NHLBI Mammalian Genotyping Service for conducting the genome-wide scan. Work of the NHLBI Mammalian Genotyping Service was supported in part by NIH/NHLBI POE PO1-HL41484. Dr. Meigs is supported by a Career Development Award from the American Diabetes Association. Drs. Cupples and Herbert and the National Heart, Lung, and Blood Institute's Framingham Heart Study are supported by a contract from the National Institutes of Health (NIH/NHLBI contract NO1-HC-38083).

\section{References}

1. American Diabetes Association (1997) Report of the Expert Committee on the Diagnosis and Classification of Diabetes Mellitus [comment]. Diabetes Care. 20: 11831197

2. Saad MF, Knowler WC, Pettitt DJ, Nelson RG, Charles MA, Bennett PH (1991) A two-step model for development of non-insulin dependent diabetes mellitus. Am J Med 90:229-235

3. Pratley RE, Weyer C (2001) The role of impaired early insulin secretion in the pathogenesis of Type 2 diabetes mellitus. Diabetologia 44:929-945

4. Pratley RE, Thompson DB, Prochazka M et al. (1998) An autosomal genomic scan for loci linked to prediabetic phenotypes in Pima Indians. J Clin Invest 101:1757-1764

5. Mitchell BD, Cole SA, Hsueh WC et al. (2000) Linkage of serum insulin concentrations to chromosome $3 p$ in Mexican Americans. Diabetes 49:513-516

6. Watanabe RM, Ghosh S, Langefeld CD et al. (2000) The Finland-United States Investigation of Non-Insulin-Dependent Diabetes Mellitus Genetics (FUSION) Study. II. An autosomal genome scan for diabetes-related quantitativetrait loci. Am J Hum Genet 67:1186-1200

7. Kannel WB, Feinleib M, Mcnamara JR, Garrison RJ, Castelli WP (1979) An investigation of coronary heart disease in families: the Framingham Offspring Study. Am J Epidemiol 110:281-290

8. World Health Organization (1980) WHO Expert Committee on Diabetes mellitus: Second Report World Health Organization, Geneva

9. Kannel WB, Sorlie P (1979) Some health benefits of physical activity: the Framingham Study. Arch Intern Med 139:857-861 
10. Matthews DR, Hosker JP, Rudenski AS, Naylor BA, Treacher DF, Turner RC (1985) Homeostasis model assessment: insulin resistance and beta-cell function from fasting plasma glucose and insulin concentrations in man. Diabetologia 28:412-419

11. Howard G, Bergman R, Wagenknecht LE et al. (1998) Ability of alternative indices of insulin sensitivity to predict cardiovascular risk: comparison with the "minimal model”. Insulin Resistance Atherosclerosis Study (IRAS) Investigators. Ann Epidemiol 8:358-369

12. Bonora E, Targher G, Alberiche M et al. (2000) Homeostasis model assessment closely mirrors the glucose clamp technique in the assessment of insulin sensitivity. Diabetes Care: 23:57-63

13. Haffner SMMH, Gaskill SP, Stern MP (1995) Decreased insulin secretion and increased insulin resistance are independently related to the 7-year risk of NIDDM in MexicanAmericans. Diabetes 44:1386-1391

14. Haffner SM, Kennedy E, Gonzalez C, Stern MP, Miettinen $\mathrm{H}$ (1996) A prospective analysis of the HOMA model. The Mexico City Diabetes Study. Diabetes Care 19:1138-1141

15. Haffner SM, Miettinen H, Gaskill SP, Stern MP (1996) Decreased insulin action and insulin secretion predict the development of impaired glucose tolerance. Diabetologia 39:1201-1207

16. Weber JL, May PE (1989) Abundant class of human DNA polymorphisms which can be typed using the polymerase chain reaction. Am J Hum Genet 44:388-396

17. Yuan B, Vaske D, Weber JL, Beck J, Sheffield VC (1997) Improved set of short-tandem-repeat polymorphisms for screening the human genome. Am J Hum Genet 60:459-460

18. Fulker DW, Cherny SS, Cardon LR (1995) Multipoint interval mapping of quantitative trait loci, using sib pairs. Am J Hum Genet 56:1224-1233

19. Lander E, Kruglyak L (1995) Genetic dissection of complex traits: guidelines for interpreting and reporting linkage results. Nat Genet 11:241-247

20. Knowler WC, Pettitt DJ, Savage PJ, Bennett PH (1981) Diabetes incidence in Pima Indians: contributions of obesity and parental diabetes. Am J Epidemiol 113:144-156

21. Looker H C, Knowler W C, Hanson R L (2001) Changes in $\mathrm{BMI}$ and weight before and after the development of type 2 diabetes. Diabetes Care 24:1917-1922

22. Colditz GA, Willett WC, Stampfer MJ et al. (1990) Weight as a risk factor for clinical diabetes in women. Am J Epidemiol 132:501-513

23. Wannamethee SG, Shaper AG (1999) Weight change and duration of overweight and obesity in the incidence of type 2 diabetes. Diabetes Care 22:1266-1272

24. Brancati FL, Wang NY, Mead LA, Liang KY, Klag MJ (1999) Body weight patterns from 20 to 49 years of age and subsequent risk for diabetes mellitus: the Johns Hopkins Precursors Study. Arch Intern Med 159:957-963

25. Campbell PJ, Carlson MG (1993) Impact of obesity on insulin action in NIDDM. Diabetes 42:405-410

26. Elbein SC, Wegner K, Kahn SE (2000) Reduced beta-cell compensation to the insulin resistance associated with obesity in members of caucasian familial type 2 diabetic kindreds. Diabetes Care 23:221-227
27. Hotamisligil GS, Shargill NS, Spiegelman BM (1993) Adipose expression of tumor necrosis factor-alpha: direct role in obesity-linked insulin resistance. Science 259:87-91

28. Boden G (1997) Role of fatty acids in the pathogenesis of insulin resistance and NIDDM [Review]. Diabetes 46:3-10

29. Pradhan AD, Manson JE, Rifai N, Buring JE, Ridker PM (2001) C-reactive protein, interleukin 6, and risk of developing type 2 diabetes mellitus. JAMA 286:327-334

30. Steppan CM, Bailey ST, Bhat S et al. (2001) The hormone resistin links obesity to diabetes. Nature 409:307-312

31. Hotta K, Funahashi T, Bodkin NL et al. (2001) Circulating concentrations of the adipocyte protein adiponectin are decreased in parallel with reduced insulin sensitivity during the progression to type 2 diabetes in rhesus monkeys. Diabetes 50:1126-1133

32. Stunkard AJ, Harris JR, Pedersen NL, Mcclearn GE (1990) The body-mass index of twins who have been reared apart. N Engl J Med 322:1483-1487

33. Chagnon YC, Perusse L, Weisnagel SJ, Rankinen T, Bouchard C (2000) The human obesity gene map: the 1999 update. Obes Res 8:89-117

34. Hanson RL, Ehm MG, Pettitt DJ et al. (1998) An autosomal genomic scan for loci linked to type II diabetes mellitus and body-mass index in Pima Indians. Am J Hum Genet 63:1130-1138

35. Mitchell BD, Kammerer CM, Mahaney MC et al. (1996) Genetic analysis of the IRS. Pleiotropic effects of genes influencing insulin levels on lipoprotein and obesity measures. Arterioscler Thromb Vasc Biol 16:281-288

36. Hong Y, Despres JP, Rice T et al. (2000) Evidence of pleiotropic loci for fasting insulin, total fat mass, and abdominal visceral fat in a sedentary population: the HERITAGE family study. Obes Res 8:151-159

37. Kissebah AH, Sonnenberg GE, Myklebust J et al. (2000) Quantitative trait loci on chromosomes 3 and 17 influence phenotypes of the metabolic syndrome. Proc Natl Acad Sci USA 97:14478-14483

38. Norman RA, Thompson DB, Foroud T et al. (1997) Genomewide search for genes influencing percent body fat in Pima Indians: suggestive linkage at chromosome 11q21-q22. Pima Diabetes Gene Group. Am J Hum Genet 60:166-173

39. Eckenrode S, Marron MP, Nicholls R et al. (2000) Finemapping of the type 1 diabetes locus(IDDM4) on chromosome $11 \mathrm{q}$ and evaluation of two candidate genes (FADD and GALN) by affected sibpair and linkage-disequilibrium analyses. Hum Genet 106:14-18

40. Yip RG, Wolfe MM (2000) GIP biology and fat metabolism. Life Sci 66:91-103

41. Ghosh S, Watanabe RM, Valle TT et al. (2000) The Finland-United States Investigation of Non-Insulin-Dependent Diabetes Mellitus Genetics (FUSION) Study. I. An autosomal genome scan for genes that predispose to type 2 diabetes. Am J Hum Genet 67:1174-1185

42. Aguilar-Bryan L, Nichols CG, Wechsler SW et al. (1995) Cloning of the beta cell high-affinity sulfonylurea receptor: a regulator of insulin secretion. Science 268:423-426

43. Luo TH, Zhao Y, Li G et al. (2001) A genome-wide search for type II diabetes susceptibility genes in Chinese Hans. Diabetologia 44:501-506 